\title{
The Influence of Micronutrients on Oral and General Health
}

\author{
B. Willershausen 1 , A. Ross ${ }^{1}$, M. Försch ${ }^{1}$, I. Willershausen ${ }^{2}$, Ph. Mohaupt ${ }^{1}$, A. Callaway ${ }^{1}$ \\ ${ }^{1}$ Department of Operative Dentistry, University Medical Center, Johannes Gutenberg University Mainz \\ ${ }^{2}$ Institute for Dental Material Sciences and Technology, University Medical Center of the Johannes Gutenberg University Mainz,
} Germany

\begin{abstract}
Objective: The aim of the present clinical pilot study was to examine the influence of a combination of micronutrients on individuals with high stress experience. Methods: 40 healthy students (28 female, 12 male) with a mean age of $27.1 \pm 3.0$ years, experiencing high examination stress, were chosen. After approval of the ethics commission, one group of students $(n=19)$ took a combination of micronutrients (Orthomol vital $\mathrm{m} / \mathrm{f}$ ) for three months, whereas other students ( $\mathrm{n}=$ 21) served as control group. All participants underwent at the beginning and at the end of the trial a dental examination, a determination of 10 periodontal pathogens, a salivary and a blood analysis. In addition, the participants filled in a questionnaire on nutrition, quality of life and degree of stress experienced during their final examinations.

Results: The evaluation of the results, obtained at the end of the trial period, showed that for all students a slight worsening of oral hygiene and an increased consumption of unhealthy food could be observed. The intake of the micronutrients led to a slight improvement of the degree of gingival inflammation in comparison to the control group. The blood analysis showed an increase in vitamin (vitamin C, vitamin E) and zinc concentrations, and a lower increase in CRP. In the male subjects, a decrease in the serum concentrations of triglycerides $(\mathrm{p}=0.073)$ and LDL ( $\mathrm{p}=$ 0.048) was observed.

Conclusions: This pilot study shows that micronutrients, taken during periods of high stress experience, had a beneficial effect on inflammatory processes and helped reduce the level of some of the plasma lipids in males, and thus can be recommended for supplementing the diet. However, additional studies with a higher number of subjects, also suffering from periodontal disease, are necessary to show the effect of a micronutrient supplementation more clearly.
\end{abstract}

Key words: micronutrients, blood analysis, gingivitis, dental examination, final examination stress

\section{INTRODUCTION}

Sound nutritional habits and a sufficient supply of essential vitamins and minerals are of considerable importance for oral health. For example, in the case of inflammatory processes a fast immune response is im- portant, which can only take place effectively when there is a sufficient supply of food and micronutrients to the body [1]. Diseases of the periodontium, like gingivitis and periodontitis, are local or generalized infections, which usually result in a local inflammatory reaction. Periodontal disease is strongly associated with the bacterial microflora present in subgingival plaque [2]; in addition to these main causative parameters, there are however additional factors like diabetes or use of tobacco products, which can cause or modify the course of a periodontitis [3].

High stress exposure is also discussed as a possible factor, exerting an unfavorable influence on periodontal health. Although stress is generally judged to be a highly personal state, which is difficult to determine, exposure to highly increased stress stimuli or permanent negative impulses can produce strains or cause various physical disorders, like e. g. tachycardia or changes in the immune system, but it can also interfere with normal learning habits [4]. A distinction has to be made between work-related, private and socio-economic stress exposure [5]. Some studies have shown that under high stress exposure the risk to develop a periodontitis increases, and that the influence on periodontal health is comparable to smoking or diabetes $[4,6]$. For example examination stress can have a negative influence on oral health, which can manifest itself in a greater plaque accumulation, local gingivitis and a change in salivary composition $[6,7]$. The various stress theories aim to explain the occurrence of stress and the resulting behavioral patterns and metabolic changes. Under high stress conditions, plaque accumulation, accompanied by gingivitis, can be considerably higher and occur more frequently during stressful periods than during stress free periods. However, one has to keep in mind that changes in periodontal health are more likely to be caused by changes in patient behavior than by metabolic changes [4].

The total antioxidant status can be used to measure the oxidative stress of a patient. By means of the TAS test, the ratio of free radicals to various antioxidants can be determined. The total antioxidant status in stressed patients is frequently altered, and, for example in patients suffering from periimplantitis, changes in the total antioxidant status are also seen [8]. A favorable change of this situation can be achieved by the intake of nutritional supplements and combinations of micronutrients. An improvement of the total antioxi- 
dant status can be detected after supplementation with antioxidants, but also after a successful conventional periodontal therapy [9]. The consumption of dietetic foods and the intake of combinations of micronutrients, but also of "functional foods", have clearly become more popular over the past years. The beneficial effect of vitamins on the body as a whole has long been known, and there are numerous studies dealing with the effects and functions of the vitamins for the human body. However, only a few studies investigate the influence of vitamins or micronutrients on oral health $[3,10]$.

Vitamin $C$ is essential for the formation and maturation of collagen, and for the integrity of connective and osteoid tissues, and dentine. In addition, it is also necessary for maintaining the proper functions of the immune system. A deficiency in vitamin $\mathrm{C}$ can lead to scurvy or interfere with normal wound healing [11]. In Europe, vitamin $\mathrm{C}$ has been added to many foods or it is already present as a natural ingredient, so that a vitamin $\mathrm{C}$ deficiency is seen relatively rarely in industrialized societies.

The vitamin B complex comprises eight different vitamins, which differ considerably, both in their chemical composition and also in their pharmacological properties. The B vitamins are needed as important precursors for various coenzymes in metabolic processes [11].

Vitamin D regulates the calcium metabolism of the human body and maintains sufficient levels of calcium and phosphate in the serum for the mineralization of the bones. At present, a deficient supply of vitamin D is frequently seen in the German population. In a study by Garcia et al. [12] a beneficial effect on the course of a chronic periodontitis was seen after a year of vitamin $\mathrm{D}$ and calcium supplementation.

Also the mineral magnesium is of major importance for the energy metabolism. In addition to causing deficiency symptoms, like for example muscle cramps and muscle twitching, a magnesium deficiency can also be associated with the onset and progression of periodontal disease [13]. In contrast, an iron deficiency resulting in iron-deficiency anemia, which is often seen in young women, seems to have little influence on oral health [14].

The importance of a balanced diet or the consumption of dietetic foods or micronutrients for the body composition, has been shown in numerous studies, and is reflected in the recommendations of various professional societies. However, there are only few studies, which have examined the possible influence of a supplemented balanced diet and not just of one vitamin complex or a single trace element / mineral on oral health. In the present pilot study, the possible influence of a micronutrient combination on various parameters of oral and systemic health was to be studied during a period of high stress exposure.

\section{Material AND Methods}

Dental students in their last term participated in the present study immediately prior to their final examinations (phase of high stress). Exclusion criteria were serious general or systemic diseases, pregnancy or long- term intake of antibiotics. After the approval of the ethics commission (number: 837.015.10 / 7024) was obtained, a total of 42 healthy young subjects were enrolled in the study. All subjects were informed in detail, both orally and in writing, about the planned trial; written informed consent to participate in the trial was obtained from all subjects.

The students were assigned to two groups, using a randomization protocol: one group $(\mathrm{n}=21)$ served as control and didn't receive any dietary supplements, the subjects in the other group $(n=21)$ received a combination of micronutrients (Orthomol vital f/m; Orthomol pharmazeutische Vertriebs $\mathrm{GmbH}$, Langenfeld, Germany), which they were asked to take daily according to the instructions, over a period of 3 months. In contrast to the micronutrient preparation for male subjects, the one for female subjects contained calcium as an additive and only half the amount of omega3 fatty acids. The subjects in both groups were not permitted to take any additional vitamins or nutritional supplements during the course of this trial. They were supposed to continue their daily oral hygiene measures as usual, and they received no specific instructions concerning the use of particular tooth pastes or tooth brushes.

The subjects answered questions concerning their dietary habits, and filled in a short questionnaire (a WHO form) with regard to their presently perceived quality of live.

All subjects underwent a dental assessment, and had a blood sample taken, first at the beginning (at baseline, phase of high stress), and again at the end of this trial (phase of relaxation). After 3 months (end of the trial), only 19 subjects from the group taking micronutrients, and all 21 subjects from the control group could be reached for the examinations at the end of the study, and only the data from these 40 subjects were included in this study. One subject from the control group missed his appointment for the second dental examination, and was therefore not included in the analyses of API and SBI $[15,16]$.

\section{Dental Assessment}

The dental assessment included the determination of the DMFT index, the degree of gingival inflammation (SBI) [15], the approximal plaque index (API) [16], the salivary flow rate and $\mathrm{pH}$. After the determination of the respective pocket depths, a microbiological analysis of the subgingival plaque from selected periodontal sites (probing depth $>3 \mathrm{~mm}$, pooled samples) was performed by an external company (ParoCheck 10, NeuroProfile, Regensburg, Germany). After the removal of supragingival plaque and drying of the tooth with a cotton roll, in each quadrant a sample was taken from the deepest probing sites with a paper point, and the presence or absence of 10 different bacteria, which have been implicated in periodontal diseases, (Porphyromonas gingivalis, Tannerella forsytbia, Treponema denticola, Campylobacter rectus/showae, Fusobacterium nucleatum ssp., Parvimonas micra, Prevotella intermedia, Aggregatibacter actinomycetemcomitans, Eikenella corrodens, Actinomyces viscosus), was determined. The dental assessment was followed by an 
analysis of the saliva. The salivary flow rate and $\mathrm{pH}$, and the total antioxidant status (TAS) were determined. Each subject chewed on a piece of paraffin for five minutes, and the saliva was collected in a graduated plastic beaker. The amount of collected saliva was recorded in $\mathrm{ml}$, and to calculate the salivary flow rate, it was divided by five and expressed as $\mathrm{ml} / \mathrm{min}$. The salivary $\mathrm{pH}$ was measured by means of a portable $\mathrm{pH}$ Meter with an accuracy of $\pm \mathrm{pH} 0.01$, equipped with a microelectrode (HI9224, Novodirect, Kehl, Germany). The TAS (total antioxidant status) was determined spectrophotometrically (Shimadzu UV-1202/ UV-VIS, Shimadzu Kyoto, Japan) by means of a colorimetric assay, using a test kit (Randox Laboratories, Krefeld, Germany).

\section{BLOOD ANALYSIS}

From each subject a blood sample (approximately 15 $\mathrm{ml}$ ) was taken by a certified medical technician at the beginning (baseline) and at the end of the study. The analyses of the samples were performed by an external laboratory (Bioscentia Labor, Ingelheim, Germany). The concentrations of homocysteine, vitamin C, D and E, malondialdehyde, zinc, C-reactive protein (CRP), high density lipoprotein cholesterol (HDL) and low density lipoprotein cholesterol (LDL), triglycerides and cortisol were determined.

\section{Statistical Data Analysis}

The statistical analysis of the data was carried out with the program SPSS for medical statistics (17.0 for Windows, Chicago, IL, USA) in collaboration with the Institute of Medical Biostatistics, Epidemiology and Informatics of the University Medical Center of the Johannes Gutenberg University Mainz. For the descriptive analysis of the data, categorial variables are presented as absolute und relative frequencies, and for the numerical variables minima, means and standard deviations, medians and maxima were calculated. To statistically analyze the differences between the two groups, the Chi-square test and the Mann-Whitney U-Test were used, and the significance level was set at $\mathrm{p}<$ 0.05 .

\section{RESULTS}

Of the total of 42 dental students, recruited immediately prior to their final examination and randomly assigned to one of two groups, only 19 subjects (5 male, 14 female) from the group taking micronutrients, and 21 subjects ( 7 male, 14 female) from the control group could be reached after 3 months for the examinations at the end of the study. The age of the subjects ranged from $24-37$ years (mean: $27.1 \pm 3.0$ years).

At baseline, the mean API value of the 20 subjects in the control group (one subject didn't come for the dental examination) was $12.3 \pm 6.3 \%$, and after 3 months it was $17.5 \pm 9.1 \%$. Similar values were found also for the group taking micronutrients: At baseline the mean value was $11.9 \pm 8.8 \%$, and at the end it was $17.9 \pm 15.2 \%$. This indicates that all students had slightly neglected their oral hygiene during the phase of high stress exposure. The difference in API between the two groups was not statistically significant ( $\mathrm{p}=0.61)$.

The degree of gingival inflammation (SBI) changed accordingly in a similar fashion.

The worsening of oral hygiene resulted in a slightly higher SBI value (Fig. 1).

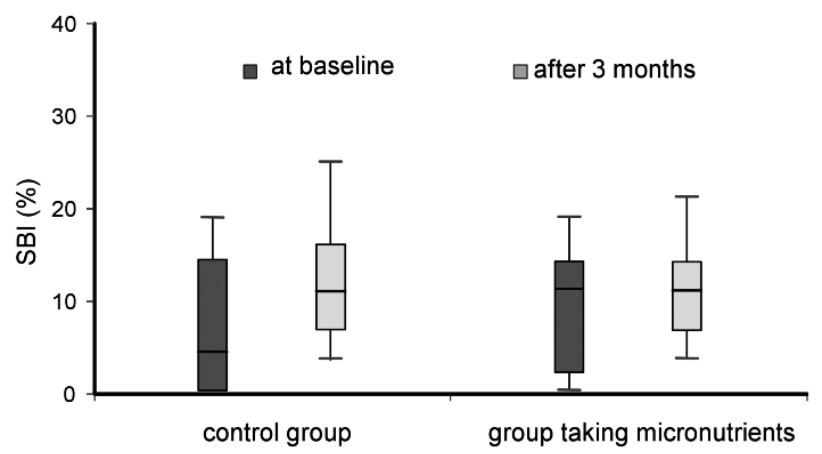

Fig. 1. Inflammatory status of the gingiva (sulcular bleeding index, SBI) in the control group $(\mathrm{n}=20)$ and the micronutrient group $(\mathrm{n}=19)$ at baseline and after 3 months. Also shown are median, minima and maxima.

The 20 subjects in the control group had at baseline a mean SBI value of $6.9 \pm 6.5 \%$ and at the end of the study a value of $11.9 \pm 6.5 \%$. A slightly, but not significantly, smaller increase in this value was found in the group taking micronutrients $(p=0.57)$. At baseline the mean SBI value was $9.2 \pm 9.0 \%$, and showed only a slight worsening to $12.6 \pm 7.9 \%$.

When the values for the salivary $\mathrm{pH}$ of the subjects in both groups were compared, no significant differences were found $(p=0.93)$. In addition, the salivary flow rate $(\mathrm{ml} / \mathrm{min})$ was determined, which plays an important role in the etiology of caries and gingivitis. Likewise, no difference could be found between the two groups $(p=0.48)$. When the oxidative stress, TAS (total antioxidant status, $\mathrm{mmol} / \mathrm{l}$ ) in whole saliva was determined, in both groups a similar decrease in value was observed after a period of 3 months, which remained, however, in the normal range. The subjects in the control group had at baseline a mean TAS value of $1.74 \pm 0.32(\mathrm{mmol} / \mathrm{l})$, and the end of the study this value was $1.0 \pm 0.41(\mathrm{mmol} / \mathrm{l})$. A somewhat smaller, but not significant decrease in TAS was found in the group taking micronutrients $(\mathrm{p}=0.43)$.

The microbiological analysis of the subgingival plaque samples yielded for both groups similar results. Samples from selected periodontal sites were tested for the presence of 10 different periodontal pathogens. In both groups Aggregatibacter actinomycetemcomitans and Porphyromonas gingivalis were not detected. The other bacterial species were found with similar frequencies and changes both in the control group and in the group taking micronutrients.

The evaluation of the two questionnaires concerning dietary habits and quality of life found similar responses in both groups. Due to the high stress experience, both groups paid less attention to eating a healthy diet, and, especially towards the end of their examinations, more often fast food und sweets were 
Table 1. Blood analysis (means $\pm \mathrm{SD}$ ) of the control group ( $\mathrm{n}=21 ; 7$ male, 14 female) and the group taking micronutrients ( $\mathrm{n}$ = 19; 5 male, 14 female), at baseline and after 3 months.

\begin{tabular}{|c|c|c|c|c|c|}
\hline \multirow[b]{2}{*}{ Parameter } & \multicolumn{2}{|c|}{ control group } & \multicolumn{2}{|c|}{ group taking micronutrients } & \multirow[t]{2}{*}{ p-value } \\
\hline & at baseline & after 3 months & at baseline & after 3 months & \\
\hline Zinc ( $\mu \mathrm{g} / \mathrm{dl}$ serum $)$ & $79.4 \pm 13.9$ & $82.8 \pm 18.5$ & $72.3 \pm 12.5$ & $90.2 \pm 29.3$ & $\mathrm{p}=0.13$ \\
\hline CRP (mg/l serum) & $2.4 \pm 4.9$ & $4.9 \pm 10.8$ & $3.3 \pm 4.8$ & $3.9 \pm 9.4$ & $\mathrm{p}=0.059$ \\
\hline Cortisol ( $\mu \mathrm{g} / \mathrm{dl}$ serum) & $19.5 \pm 5.9$ & $18.0 \pm 5.8$ & $18.9 \pm 6.6$ & $17.5 \pm 8.9$ & $\mathrm{p}=0.13$ \\
\hline Homocysteine ( $\mu \mathrm{mol} / \mathrm{l}$ blood $)$ & $14.0 \pm 2.8$ & $11.3 \pm 1.9$ & $12.5 \pm 3.1$ & $9.8 \pm 2.2$ & $\mathrm{p}=0.85$ \\
\hline Vitamin D (ng/ml serum) & $15.7 \pm 8.7$ & $25.6 \pm 10.2$ & $17.2 \pm 6.5$ & $25.6 \pm 6.2$ & $\mathrm{p}=0.65$ \\
\hline Vitamin C (mg/l plasma) & $7.6 \pm 2.8$ & $9.0 \pm 2.5$ & $8.0 \pm 2.2$ & $11.3 \pm 3.5$ & $\mathrm{p}=0.49$ \\
\hline Vitamin E ( $\mu \mathrm{g} / \mathrm{ml}$ serum $)$ & $15.5 \pm 1.7$ & $14.6 \pm 2.2$ & $14.6 \pm 3.8$ & $16.2 \pm 3.3$ & $\mathrm{p}=0.055$ \\
\hline Malondialdehyde ( $\mu \mathrm{mol} / 1$ plasma) & $5.5 \pm 2.7$ & $2.1 \pm 0.5$ & $5.7 \pm 3.8$ & $2.4 \pm 0.8$ & $\mathrm{p}=0.97$ \\
\hline Triglycerides (m) (mg/dl serum) & $117.4 \pm 71.8$ & $171.2 \pm 85.8$ & $141.4 \pm 77.0$ & $134.2 \pm 103.9$ & $\mathrm{p}=0.073$ \\
\hline HDL (m) (mg/dl serum) & $57.3 \pm 13.7$ & $52.4 \pm 12.0$ & $52.4 \pm 11.2$ & $52.2 \pm 8.2$ & $\mathrm{p}=0.53$ \\
\hline LDL (m) (mg/dl serum) & $108.4 \pm 24.1$ & $107.4 \pm 20.3$ & $137.4 \pm 72.2$ & $114.6 \pm 58.7$ & $\mathrm{p}=0.048^{*}$ \\
\hline Triglycerides (f) (mg/dl serum) & $113.6 \pm 38.9$ & $105.3 \pm 35.1$ & $110.6 \pm 55.7$ & $102.0 \pm 43.2$ & $\mathrm{p}=0.45$ \\
\hline HDL (f) (mg/dl serum) & $71.1 \pm 13.1$ & $67.8 \pm 13.8$ & $72.2 \pm 17.2$ & $69.3 \pm 11.3$ & $\mathrm{p}=0.94$ \\
\hline LDL (f) (mg/dl serum) & $104.3 \pm 22.1$ & $94.5 \pm 24.8$ & $84.3 \pm 18.1$ & $78.3 \pm 14.8$ & $\mathrm{p}=0.21$ \\
\hline
\end{tabular}

consumed. Due to the very high stress experienced by the examinations, the intake of a combination of micronutrients, didn't lead to any major changes with regard to dietary habits and experienced quality of life.

The blood analysis resulted in minor differences between the subjects in the control group and those, taking micronutrients, so that a beneficial effect of the intake of a combination of micronutrients was shown (Table 1).

To give an example, the control group had at baseline for zinc a mean value of $79.4 \pm 13.9$ and at the end of the study of $82.8 \pm 18.5$ ( $\mu \mathrm{g} / \mathrm{dl}$ plasma), whereas in the group taking micronutrients the concentration had increased from $72.3 \pm 12.5$ at baseline to $90.2 \pm 29.3(\mu \mathrm{g} / \mathrm{dl}$ plasma) after 3 months $(\mathrm{p}=$ 0.13 ). Likewise, in the group taking micronutrients the concentrations of the vitamins $\mathrm{C}$ and $\mathrm{E}$ had increased after three months.

Due to the increase in stress experience during the examinations, the CRP values increased slightly in both groups; however, the CRP value, as markers for possible inflammatory processes, increased somewhat less in the group taking micronutrients $(p=0.059)$.

In the male subjects in the micronutrient group, a decrease in the serum concentrations of triglycerides from $141.4 \pm 77.0$ to $134.2 \pm 103.9 \mathrm{mg} / \mathrm{dl}$ serum $(\mathrm{p}=$ 0.073 , and in LDL from $137.4 \pm 72.2$ to $114.6 \pm 58.7$ $\mathrm{mg} / \mathrm{dl}(\mathrm{p}=0.048)$ was observed.

\section{Discussion}

Nutrients play an important regulatory role in preserving health of the human body and of all metabolically active tissues. Micronutrients, vitamins and antioxidants play an essential role for constant regenerative processes, for coping with oxidative stress, and also for adequate immune responses [17, 18]. In oral health, undernutrition or malnutrition concerning certain food components can lead to defects of the den- tal hard tissues, the oral mucosa and the periodontium $[1,19]$.

Sound dietary habits can also contribute considerably to the prevention of certain oral tumors [20]. It was shown that in persons, consuming sufficient amounts of fruit, whole grain foods and vegetables, the risk to develop oral or pharyngeal tumors can be lowered by $40-80 \%$, in comparison with persons, which consume these types of foods considerably less frequently [21, 22].

In several epidemiological studies, relationships of periodontal inflammatory processes with systemic diseases like diabetes mellitus, atherosclerosis, rheumatic diseases and other disorders with chronic inflammatory processes could be shown [23, 24].

Especially in the case of periodontal inflammation, nutrition-based interventions as a part of a possible therapy concept are discussed [25]. While the medical literature contains many reports on the effectiveness of various nutrients and vitamins on wound healing, considerably fewer studies with regard to the importance of micronutrients and periodontal diseases can be found. For example, two studies by Nishida et al. $[26,27]$ could show a weak but significant relation of the intake of vitamin $\mathrm{C}$ and calcium and a reduced susceptibility to periodontal diseases. In the present clinical study on micronutrients, a slight beneficial effect of an optimal supply of nutrients on parameters of inflammatory processes could also be demonstrated.

It is certainly of major importance that the periodontal state cannot be looked at independently of general health, and that in consequence a specific diet for oral health cannot be discussed as such. To find a definite relationship between nutrition and oral health, further clinical studies are needed, and patients with or without an optimal nutritional state would have to be compared and examined accordingly. In summary, it can be assumed that an additional intake of nutritional 
supplements will have a slightly positive effect and will generally not cause any side effects. Further controlled clinical studies will certainly be needed to show, for example, that specific foods and combinations of micronutrients can be used to improve the response to therapies, and can complement treatment concepts.

\section{REFERENCES}

1. Moynihan PJ. The role of diet and nutrition in the etiology and prevention of oral diseases. Bull World Health Organ. 2005; 83: 694-9.

2. Loesche WJ. Bacterial mediators in periodontal disease. Clin Infect Dis. 1993; 16: 203-10.

3. Logan EI. Dietary influences on periodontal health in dogs and cats. Vet Clin North Am Small Anim Pract. 2006; 36: 1385-401.

4. Rosania AE, Low KG, McCormick CM, Rosania DA. Stress, depression, cortisol, and periodontal disease. J Periodontol. 2009; 80: 260-6.

5. Genco RJ, Ho AW, Kopman J, Grossi SG, Dunford RG, Tedesco LA. Models to evaluate the role of stress in periodontal disease. Ann Periodontol. 1998; 3: 288-302.

6. Ng SK, Keung Leung W. A community study on the relationship between stress, coping, affective dispositions and periodontal attachment loss. Community Dent Oral Epidemiol. 2006; 34: 252-66.

7. Johannsen A, Bjurshammar N, Gustafsson A. The influence of academic stress on gingival inflammation. Int J Dent Hyg. 2010; 8: 22-7.

8. Chapple IL. Oxidative stress, nutrition and neutrogenomics in periodontal health and disease. Int J Dent Hyg. 2006; 4: 15-21.

9. Abou Sulaiman AE, Shehadeh RM. Assessment of total antioxidant capacity and the use of vitamin $\mathrm{C}$ in the treatment of non-smokers with chronic periodontitis. J Periodontol. 2010; 81: 1547-54.

10. Jenzsch A, Eick S, Rassoul F, Purschwitz R, Jentsch H. Nutritional intervention in patients with periodontal disease: clinical, immunological and microbiological variables during 12 months. Br J Nutr. 2009; 101: 879-85.

11. Neiva RF, Steigenga J, Al-Shammari KF, Wang HL. Effects of specific nutrients on periodontal disease onset, progression and treatment. J Clin Periodontol. 2003; 30: 579-89.

12. Garcia MN, Hildebolt CF, Miley DD, Dixon DA, Couture RA, Spearie CL, Langenwalter EM, Shannon WD, Deych E, Mueller C, Civitelli R. One-year effects of vitamin $\mathrm{d}$ and calcium supplementation on chronic periodontitis. J Periodontol. 2011; 82: 25-32.

13. Meisel P, Schwahn C, Luedemann J, John U, Kroemer HK, Kocher T. Magnesium deficiency is associated with periodontal disease. J Dent Res. 2005; 84: 937-41.

14. Enhos S, Duran I, Erdem S, Buyukbas S. Relationship between iron-deficiency anemia and periodontal status in female patients. J Periodontol. 2009; 80: 1750-55.

15. Löe H, Silness J. Periodontal disease in pregnancy. I. Prevalence and severity. Acta Odontol Scan. 1963; 21: 533-51.
16. Lange DE. Die Anwendung von Indices zur Diagnostik der Perodontopathien. Dtsch Zahnärztl Z. 1978; 33: 8-11.

17. Chapple IL, Brock GR, Milward MR, Ling N, Matthews JB. Compromised GCF total antioxidant capacity in periodontitis: cause or effect? J Clin Periodontol. 2007; 42: 103-10.

18. Enwonwu CO, Phillips RS, Falkler WA. Nutrition and oral infectious diseases: state of the science. Compend Contin Educ Dent. 2002; 23: 431-6.

19. Chen M, Andersen RM, Barmes DE, Leclerq M-H, Lyttle SC. Comparing oral health systems. A Second International Collaborative Study. Geneva: World health Organization; 1997.

20. Morse DE. Oral and pharyngeal cancer. In. TougerDecker R, Sirois DA, Mobley CC, editors. Nutrition and oral medicine. New Jersey: Humana Press; 2004: 205-21.

21. Franceschi S, Favero A, Conti E, Talamini R, Volpe R, Negri E, Barzan L, La Vecchia C. Food groups, oils and butter and cancer of the oral cavity and pharynx. Br J Cancer. 1999; 80: 614-20.

22. McLaughlin JK, Gridley G, Block G, Winn DM, PrestonMartin S, Schoenberg JB, Greenberg RS, Stemhagen A, Austin DF, Ershow AG, Blot WJ Fraumeni JF. Dietary factors in oral and pharyngeal cancer. J Natl Cancer Inst. 1988; 80: 1237-43.

23. Beck JD, Offenbacher S. Systemic effects of periodontitis: epidemiology of periodontal disease and cardiovascular disease. J Periodontol. 2005; 76: 2089-100.

24. Taylor GW. Bidirectional interrelationships between diabetes and periodontal diseases: an epidemiologic perspective. Ann Periodontol. 2001; 6: 99-112.

25. Chapple IL. Potential mechanisms underpinning the nutritional modulation of periodontal inflammation. J Am Dent Assoc. 2009; 140: 178-84.

26. Nishida M, Grossi SG, Dunford RG, Ho AW, Trevisan $\mathrm{M}$, Genco RJ. Calcium and the risk for periodontal disease. J Periodontol. 2000; 71: 1057-66.

27. Nishida M, Grossi SG, Dunford RG, Ho AW, Trevisan M, Genco RJ. Dietary vitamin $\mathrm{C}$ and the risk for periodontal disease. J Periodontol. 2000; 71: 1215-23.

Received: June 30, 2011 / Accepted: July 11, 2011

Address for correspondence:

Prof. Brita Willershausen

Department of Operative Dentistry

University Medical Center

Johannes Gutenberg-University Mainz

Augustusplatz 2

55131 Mainz

Germany

Tel.: $\quad+49(0) 6131-177246$

Fax: +49(0)6131-173406

E-Mail: willersh@uni-mainz.de 